\title{
CARNE CAPRINA DE ANIMAIS MESTIÇOS: ESTUDOS DO PERFIL AROMÁTICO ${ }^{1}$
}

\author{
M. S. MADRUGA ${ }^{2, *}$, J. G SOUZA², S. G. B. ARRUDA², Narendra NARAIN ${ }^{2}$
}

\begin{abstract}
RESUMO
Análises do perfil aromático da carne caprina cozida de animais mestiços foram realizadas utilizando-se animais castrados e inteiros, abatidos com idades de 175, 220, 265 e 310 dias. O perfil aromático da carne caprina foi constituído por 108 voláteis, sendo que 69 foram positivamente identificados e 39 parcialmente caracterizados utilizando-se análises de CG-EM. O perfil aromático da carne caprina foi formado por hidrocarbonetos alifáticos e alicíclicos, aldeídos, compostos benzênicos, álcoois, cetonas, terpenóides, ésteres e compostos heterocíclicos sulfurados, hexadecanal, benzeno, heptano e octadecanal foram os voláteis que apresentaram os maiores índices de abundância relativa. Nas análises quantitativa e qualitativa observaram-se uma predominância de voláteis nos extratos de carne de caprinos castrados. O número total de voláteis e a abundância relativa das diferentes classes de compostos não foram claramente afetados pelo fator idade de abate

Palavras-chave: carne caprina; voláteis; idade de abate; castração.
\end{abstract}

\section{SUMMARY}

GOAT MEAT OF “MESTIÇO” ANIMALS: VOLATILE PROFILE ANALYSIS. The volatile profile of cooked goat meat was analysed using meat from castrated and intact animals slaughtered at 175, 220, 265 and 310 days. A total of 108 volatiles was detected and from them 69 was identified and a further 39 were partially characterised by GC-MS. The volatile profile was composed by hydrocarbons aliphatic and alicyclic, aldehydes, benzenoid compounds, alcohols, ketones, terpenoids, esters and sulfur compounds. Hexadecanal, benzen, heptane and octadecanal were among the volatiles with highest relative abundance. In both qualitative and quantitative analyses extracts from castrated meat had higher production of volatiles. The total number and the relative abundance of different classes of compounds seemed not to be cleared affected by slaughter age factor.

Keywords: goat meat; flavours; slaughter age; castration

\section{1 - INTRODUÇÃO}

O aroma é um dos atributos mais importante da carne cozida. Dentre as carnes brancas e vermelhas, a carne bovina tem sido a mais pesquisada, do ponto de vista aromático. Considerando-se as carnes ovinas e caprinas, que são bastante semelhantes organolépticamente, observa-se na literatura que os trabalhos realizados têm enfocado principalmente os voláteis da carne ovina, tanto no músculo como no tecido adiposo [4, 19, 21, 22, 23, 24], sendo que quase nada tem sido reportado sobre o aroma da carne caprina [14].

Uma vez que carne ovina ou caprina cozidas apresentam um aroma bastante característico e diferente das demais carnes vermelhas [4], vários estudos têm sido direcionados na identificação dos compostos voláteis responsáveis por este odor característico destas espécies, observando-se que existe uma grande evidência de que certos ácidos graxos de cadeia ramificada sejam os responsáveis pelo aroma característico da carne ovina e/ou caprina [16].

YOUNG et al. [23] reportaram que análises de componentes principais identificaram os ácidos graxos de cadeias ramificadas, como os principais voláteis do aroma característico da carne ovina, destacando-se dentre estes o ácido 4-metil nonanoíco. SUTHERLAND \& AMES [22] também indicaram os ácidos graxos 4-metil octanóico 1. Recebido para publicação em 14/05/2001. Aceito para publicação em 17/02/2003 (000634).

2 Departamento de Tecnologia Quimica e de Alimentos (DTQA/CT/UFPB). Campus I. João Pessoa/PB. E-mail:msmadruga@uol.com.br

* A quem a correspondência deve ser enviada. e 4-metil nonanóico como sendo os responsáveis pelo "flavour" característicos de ovinos.

É sabido, pelo gosto popular, que a carne caprina, principalmente de animal macho adulto, apresenta um aroma forte, distinto e desagradável, caracterizado como "caprino", porém evidências científicas que justifiquem este aroma são inconclusivas [12, 14].

KIM \& LINDSAY [7] reportaram que na carne caprina das raças "Korean black" e "American white" os ácidos graxos 4-metil octanóico e 4-etil octanoíco foram os principais voláteis do aroma "caprino", sendo que o "flavour" da carne caprina e ovina estava associado respectivamente com a presença dos ácidos graxos 4-etil-octanoíco e 4-metil-octanoico. WONG et al. [24] propuseram que certos ácidos graxos com cadeias ramificadas com grupo metil, presente na gordura subcutânea dos caprinos, seriam os componentes diretamente responsáveis pelo odor "caprino", e confirmaram esta hipótese através de análise sensorial, na qual o odor "caprino" foi relacionado com a presença do ácido 4-metil octanóico. Posteriormente, HA \& LINDSAY [6] relacionaram este aroma com os componentes fenólicos isolados do tecido adiposo da carne caprina.

BRENNAND [2] estudando as propriedades aromáticas e os valores de "threshold" dos ácidos graxos de cadeias ramificadas, reportaram que o ácido graxo 4-etil octanóico apresentou o menor valor de "threshold" dentre os vinte e três ácidos analisados, observando ainda que ácidos graxos contendo ramificações na posição "4", apresentaram odores característicos de "caprino/ovino", assim como ácidos graxos contendo cadeias com oito átomos de carbono. 
Nesta pesquisa, o perfil aromático da carne cozida de caprinos castrados e/ou inteiros abatidos com diferentes idades foi pesquisado, objetivando entender a influência destes fatores na composição de voláteis da mesma proveniente de animais mestiços.

\section{2 - MATERIAL E MÉTODOS}

\section{1 - Material}

No estudo realizado o material utilizado constou de dezesseis caprinos machos mestiços de cruzas das raças Crioulo X Anglo Nubiano ou Saaneno. Os caprinos foram divididos em dois grupos de oito animais cada um, sendo que oito animais foram castrados através de remoção dos testículos ao completarem 105 dias de nascidos e oito foram deixados inteiros. Todos os caprinos foram criados em regime de confinamento, com controle quinzenal de idade e peso, alimentados com capim elefante e ração. Foram realizados quatro abates consecutivos, em grupo de quatro animais, dois inteiros e dois castrados, observando-se intervalos de 45 dias entre eles, os quais corresponderam às idades de 175, 220, 265 e 310 dias. Os procedimentos utilizados no abate e na desossa foram os mesmos descritos por MADRUGA et al. $[11,14]$.

\section{2 - Análise instrumental dos voláteis da carne caprina cozida}

Vinte e quatro amostras foram analisadas no Laboratório de Flavour do Departamento de Tecnologia Química de Alimentos da Universidade Federal da Paraíba (UFPB), no que se refere ao perfil aromático da carne caprina cozida. Os voláteis da carne caprina foram extraídos utilizando-se a técnica de extração/destilação simultânea de voláteis em aparelho de LIKENS \& NICKERSON [8], seguido de separação e identificação em CG-EM. Todas as extrações foram realizadas em triplicatas.

\subsection{1 - Extração e destilação simultânea dos vo- láteis}

Aproximadamente $200 \mathrm{~g}$ de carne desossada foram cozidas em forno convencional por 55 min [12]. Após resfriamento a carne cozida foi transferida para o balão de extração, adicionando-se $500 \mathrm{~mL}$ de água destilada seguida de agitação. Trinta mililitros $(30 \mathrm{~mL})$ de uma mistura contendo éter etílico:pentano na proporção de 3:27 (v/v) foram transferidos para o balão de solvente com capacidade de $50 \mathrm{~mL}$. Os conteúdos de ambos os frascos foram aquecidos e o processo de extração/destilação foi seguido durante $2 \mathrm{~h}$. Após o processo de extração, o extrato aromático foi resfriado a temperatura ambiente, transferido para o aparelho de "KudernaDanish" e concentrado em um banho-maria a $40^{\circ} \mathrm{C}$ até um volume final de 3 a $5 \mathrm{~mL}$. Finalmente o extrato aromático concentrado foi transferido para ampolas de vidro e seladas com maçarico. As ampolas foram armazenadas em freezer $\mathrm{a}-15^{\circ} \mathrm{C}$, até a realização das análises em CG-EM.

\subsection{2 - Análises em cromatógrafo a gás - espectrometro de massa (CG-EM)}

Os extratos da carne caprina cozida foram analisados em CG-EM, utilizando-se um detetor de espectrometria de massa - HP 5972, acoplado a um cromatográfo a gás - HP 5890, ligado a uma "workstation" - HP 59970 (versão 3.2) (Hewlett-Packard, Bracknell, Bershire, UK). Um injetor tipo "split-splitless" mantido a $200^{\circ} \mathrm{C}$ foi utilizado para introduzir-se $1 \mu \mathrm{L}$ do extrato caprino em uma coluna capilar de sílica fundida BPX5, com $50 \mathrm{~m} \mathrm{X}$ $0,32 \mathrm{~mm}$ (d.i.) $\mathrm{X} 0,5 \mu \mathrm{m}$ de filme da fase liquida (J\&W Scientific Inc). A temperatura inicial da coluna foi de $40^{\circ} \mathrm{C}$, após $5 \mathrm{~min}$ da injeção da amostra a temperatura da coluna foi aumentada para $60^{\circ} \mathrm{C}$ a uma razão de $20^{\circ} \mathrm{C} /$ min, sendo mantida nesta temperatura por $5 \mathrm{~min}$. Finalmente programou-se um novo aumento na temperatura da coluna utilizando-se uma razão de $4^{\circ} \mathrm{C} / \mathrm{min}$, até a temperatura final de $250^{\circ} \mathrm{C}$, mantendo-se a coluna nesta temperatura por mais 10 min. Solução padrão de nalcanos homólogos $\left(\mathrm{C}_{6}-\mathrm{C}_{20}\right)$ foi analisada diariamente, antes das análises dos extratos da carne caprina, objetivando-se verificar as condições analiticas do equipamento, juntamente com o cálculo posterior do Índice de Retenção Linear (IRL) dos voláteis sendo este indice utilizado na identificação dos compostos aromáticos.

Na identificação dos voláteis utilizou-se, além do IRL, o banco de espectros da própria livraria do CG-EM, comparando-se os espectros dos voláteis dos extratos da carne caprina com os espectros de referência [1]. A quantificação dos voláteis foi obtida através da integração dos espectros de massa dos voláteis identificados.

\section{3 - RESULTADOS E DISCUSSÃO}

O perfil aromático dos extratos de carne caprina, identificados pela técnica de CG-EM, juntamente com os valores de abundância relativa de cada um dos voláteis com seus respectivos valores dos IRL, estão apresentados na Tabela 1. A abundância relativa das principais classes de compostos voláteis identificados nos extratos da carne caprina estão listadas na Tabela 2. Os 108 voláteis selecionados foram aqueles que apresentaram picos significativos nos cromatogramas. Dentre estes 69 voláteis foram positivamente identificados, através da comparação de seus espectros de massa e IRL com materiais autênticos. Dezoito voláteis tiveram sua identificação baseada na comparação dos espectros obtidos com espectros de massa publicados, considerando-se a inexistência na literatura dos IRL de compostos autênticos. Vinte e um compostos foram sugeridos através da interpretação de seus espectros de massa e comparação com espectros de compostos relacionados.

O perfil aromático da carne caprina foi formado por dez classes de compostos aromáticos, sendo estas constituídas por quarenta e um hidrocarbonetos alifáticos, dezenove aldeídos, doze hidrocarbonetos alicíclicos, nove compostos benzênicos, nove álcoois, sete cetonas, dois terpenoídes e dois ésteres. Apenas quatro compostos heterocíclicos foram detectados, sendo todos compostos 
TABELA 1. Abundância relativa dos compostos voláteis identificados em extratos de carne caprina de animais inteiros e castrados abatidos com diferentes idades.

\begin{tabular}{|c|c|c|c|c|c|}
\hline \multirow{3}{*}{ No } & \multirow{3}{*}{ Classe e Nome do Composto ${ }^{a}$} & \multicolumn{4}{|c|}{ Abundância Relativa $^{c}$} \\
\hline & & $\begin{array}{c}\text { Idade (dias) } \\
175 \text { dias }\end{array}$ & 220 dias & 265 dias & 310 dias \\
\hline & & $\% 1 C \% 11$ & $\% 2 \mathrm{C} \% 21$ & $\% 3 C \% 31$ & $\% 4 C \% 41$ \\
\hline
\end{tabular}

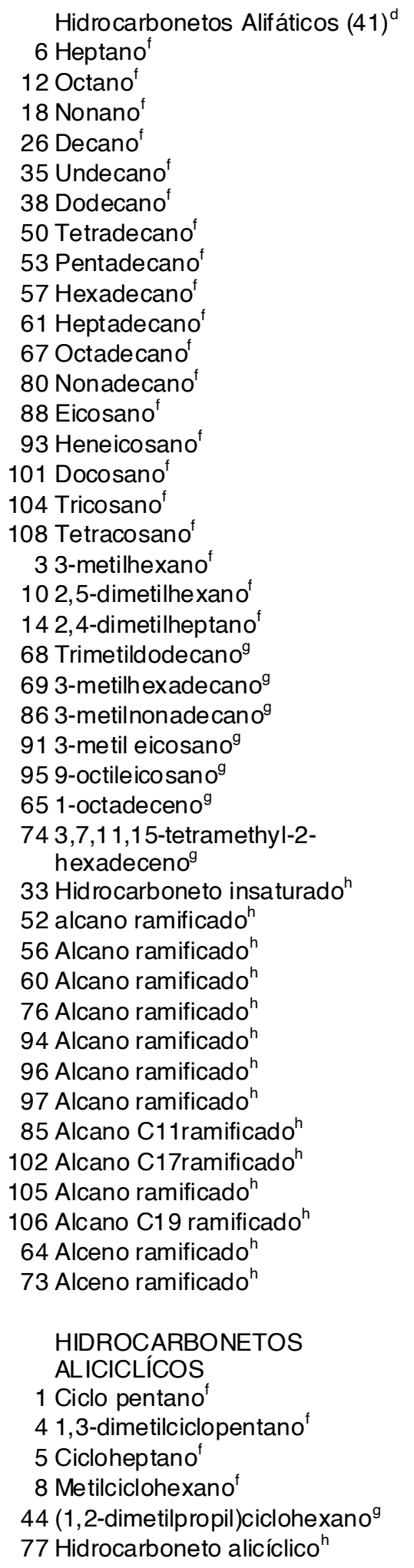

\begin{tabular}{|c|c|c|c|c|c|c|c|c|}
\hline 00 & 0 , & 6 & 5 & 15,59 & 5,22 & 8 & 0,78 & 6,42 \\
\hline 798 & 0,55 & 0,34 & 0,24 & 0,14 & 0,19 & 0,47 & 0,17 & 0,29 \\
\hline 899 & 48 & 0,22 & 0,24 & $\mathrm{Nd}^{\mathrm{e}}$ & 0,35 & 0,41 & $\mathrm{Nd}$ & 0,58 \\
\hline 998 & 0,33 & 0,15 & 0,18 & $\mathrm{Nd}$ & 0,26 & 0,32 & 0,19 & 0,46 \\
\hline 098 & 0,16 & $\mathrm{Nd}$ & 0,09 & $\mathrm{Nd}$ & 0,17 & $\mathrm{Nd}$ & 0,23 & 0,31 \\
\hline 198 & 0,05 & $\mathrm{Nd}$ & 0,03 & $\mathrm{Nd}$ & 0,20 & 0,19 & 0,07 & 0,26 \\
\hline 397 & 0,24 & 0,10 & 0,15 & 0,10 & 0,17 & $\mathrm{Nd}$ & 18 & 0,20 \\
\hline 497 & 0,60 & 0,26 & 0,60 & 0,11 & 0,23 & $\mathrm{Nd}$ & 0,27 & 0,16 \\
\hline 597 & 0,22 & 0,12 & 0,14 & 0,13 & 0,15 & $\mathrm{Nd}$ & 0,25 & 0,27 \\
\hline 696 & 0,42 & 0,34 & 0,56 & 0,49 & 0,50 & 0,55 & 0,61 & 0,50 \\
\hline 797 & 0,61 & 0,59 & 1,03 & 0,49 & 0,84 & 1,25 & 0,68 & 0,77 \\
\hline 898 & 0,43 & 0,31 & 0,30 & 0,18 & 0,42 & 0,31 & 0,43 & 0,44 \\
\hline 997 & 0,62 & 0,43 & 0,51 & 0,31 & 0,75 &, 5 & 0,96 & 1,22 \\
\hline 096 & 1,19 & 0,78 & 1,14 & 0,56 & 1,25 & 0.52 & 1,15 & 0,96 \\
\hline 196 & 2,18 & 1,63 & 1,90 & 0,85 & 2,38 & 1,65 & 1,88 & 1,37 \\
\hline 295 & 3,82 & 2,79 & 3,17 & 1,34 & 4,04 & 3.66 & 2 & 1,78 \\
\hline 397 & 5,64 & 3,59 & 3,68 & 1,85 & 2,70 & 4,90 & 3,80 & 2,73 \\
\hline 605 & $\mathrm{Nd}$ & $\mathrm{Nd}$ & 0,57 & $\mathrm{Nd}$ & $\mathrm{Nd}$ & $\mathrm{Nd}$ & 2,58 & $\mathrm{Nd}$ \\
\hline 758 & $\mathrm{Nd}$ & $\mathrm{Nd}$ & $\mathrm{Nd}$ & $\mathrm{Nd}$ & $\mathrm{Nd}$ & 0,56 & $\mathrm{Nd}$ & 0,45 \\
\hline 837 & 0,11 & $\mathrm{Nd}$ & $\mathrm{Nd}$ & $\mathrm{Nd}$ & $\mathrm{Nd}$ & $\mathrm{Nd}$ & 0,08 & 0,10 \\
\hline 799 & 0,16 & 0,24 & 0,44 & 0,17 & 0,47 & 0,71 & 0,26 & $\mathrm{Nd}$ \\
\hline $\mathrm{Nd}$ & 11 & $\mathrm{Nd}$ & $\mathrm{Nd}$ & $\mathrm{Nd}$ & $\mathrm{Nd}$ & $\mathrm{Nd}$ & 0,04 & $\mathrm{~N}$ \\
\hline 968 & $\mathrm{Nd}$ & $\mathrm{Nd}$ & 0,18 & 0,54 & 0,08 & $\mathrm{Nd}$ & 0,18 & 1,30 \\
\hline 062 & 0,18 & $\mathrm{Nd}$ & 0,37 & 0,53 & 0,09 & 0,38 & 0,60 & $\mathrm{Nd}$ \\
\hline 139 & 0,13 & $\mathrm{Nd}$ & 0,20 & $\mathrm{Nd}$ & 0,17 & $\mathrm{Nd}$ & 0,43 & 0,74 \\
\hline 1779 & 0,11 & 2,11 & 5,86 & 2,31 & 6,35 & 7,52 & 3,33 & 4,33 \\
\hline חתח & & & 221 & & & 2,80 & 1,09 & 17 \\
\hline 07 & & $\mathrm{~N}$ & , & $\mathrm{Nd}$ & $\mathrm{Nd}$ & $\mathrm{Nd}$ & & $\mathrm{N}$ \\
\hline 479 & $\mathrm{Nd}$ & $\mathrm{Nd}$ & 0,05 & $\mathrm{Nd}$ & 0,07 & $\mathrm{Nd}$ & 0,11 & 0,14 \\
\hline 1585 & 0,11 & $\mathrm{Nd}$ & 0,52 & $\mathrm{Nd}$ & 0,10 & $\mathrm{Nd}$ & 0,55 & 0,24 \\
\hline 1687 & $\mathrm{Nd}$ & $\mathrm{Nd}$ & 0,11 & 0,24 & 0,16 & $\mathrm{Nd}$ & 0,24 & 0,23 \\
\hline 1857 & 0,21 & $\mathrm{Nd}$ & 0,11 & $\mathrm{Nd}$ & 0,08 & $\mathrm{Nd}$ & 0,06 & 0,1 \\
\hline 2133 & $\mathrm{Nd}$ & 0,39 & 0,47 & 0,24 & 0,49 & 0,55 & 0,53 & 1,59 \\
\hline 144 & 0,44 & 0,35 & 0,33 & 0,14 & 0,64 & $\mathrm{Nd}$ & 0,35 & $\mathrm{Nd}$ \\
\hline 2152 & 0,37 & 0,24 & 0,10 & 0,34 & 0,30 & 0,19 & 0,41 & 0,24 \\
\hline \multirow[t]{4}{*}{1946} & 0,20 & 2,44 & 0,61 & 0,26 & 0,70 & 0,41 & 0,09 & $\mathrm{Nd}$ \\
\hline & 0,67 & 3,19 & 0,74 & 2,36 & 0,81 & 8,77 & 0,68 & $\mathrm{Nd}$ \\
\hline & 3,86 & 0,31 & 0,64 & 0,16 & 0,63 & 0,29 & $\mathrm{Nd}$ & $\mathrm{Nd}$ \\
\hline & 0,15 & 1,54 & 0,28 & 0,34 & 0,55 & 0,98 & 0,23 & 0,41 \\
\hline 749 & 0,27 & $\mathrm{Nd}$ & 0,73 & $\mathrm{Nd}$ & 0,14 & $\mathrm{Nd}$ & 0,15 & $\mathrm{Nd}$ \\
\hline 83 & $\mathrm{Nd}$ & 1,09 & 2,69 & 0,86 & 1,94 & 2,30 & 1,33 & 1,53 \\
\hline
\end{tabular}

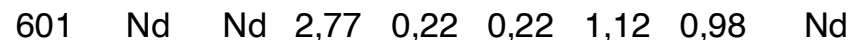

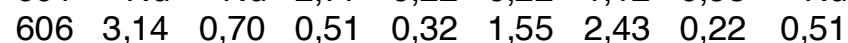
$\begin{array}{lllllllll}624 & 2,28 & 0,29 & 0,20 & \text { Nd } & 0,84 & 0,75 & 0,29 & 0,20\end{array}$

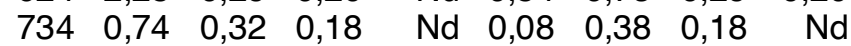
$1295 \mathrm{Nd} \quad \mathrm{Nd} \quad \mathrm{Nd} \quad \mathrm{Nd} \quad \mathrm{Nd} \quad \mathrm{Nd} 0,14 \quad \mathrm{Nd}$ $1869 \quad 0,15 \quad 0,23 \quad 0,58 \quad \mathrm{Nd} \quad 0,12 \quad \mathrm{Nd} \quad \mathrm{Nd} \quad 0,27$ 


\begin{tabular}{|c|c|c|c|c|c|c|c|c|c|c|}
\hline \multirow{4}{*}{\multicolumn{2}{|c|}{ No Classe e Nome do Composto }} & \multirow[t]{4}{*}{$\mathrm{IRL}^{\mathrm{b}}$} & \multicolumn{8}{|c|}{ Abundância Relativa $^{c}$} \\
\hline & & & \multirow{2}{*}{\multicolumn{2}{|c|}{$\begin{array}{l}\text { Idade (dias) } \\
175 \text { dias } \\
\end{array}$}} & \multirow{2}{*}{\multicolumn{2}{|c|}{220 dias }} & \multirow{2}{*}{\multicolumn{2}{|c|}{265 dias }} & \multirow{2}{*}{\multicolumn{2}{|c|}{310 dias }} \\
\hline & & & & & & & & & & \\
\hline & & & \multicolumn{2}{|c|}{$\% 1 C \% 11$} & \multicolumn{2}{|c|}{$\% 2 \mathrm{C} \% 2 \mathrm{I}$} & \multicolumn{2}{|c|}{$\% 3 C \% 31$} & \multicolumn{2}{|c|}{$\% 4 C \% 4 I$} \\
\hline & 81 Hexadecil-oxirano $^{g}$ & 1900 & 0,30 & $\mathrm{Nd}$ & 1,08 & 1,50 & 0,60 & 0,68 & 0,96 & 1,48 \\
\hline & 92 Eicosaneciclopentano $^{9}$ & 2076 & 0,29 & 0,27 & 0,33 & 0,13 & 30,30 & $\mathrm{Nd}$ & 0,56 & 0,27 \\
\hline & 03 Heneicosilciclopentano ${ }^{9}$ & & 0,35 & $\mathrm{Nd}$ & 0,40 & 0,10 & 0,05 & 0,65 & $\mathrm{Nd}$ & 1,09 \\
\hline & 00 Hidrocarboneto alicíclico ${ }^{\text {h }}$ & 2179 & 0,23 & 0,45 & 6,03 & 0,14 & $4 \quad 0,13$ & 1,72 & 0,31 & 0,54 \\
\hline & 7 1,1-dietoxi-etano 9 & 724 & 9,25 & 1,13 & 32,55 & 0,38 & 83,51 & 3,97 & 6,86 & 2,42 \\
\hline & 9 1-etoxi-pentano 9 & 751 & 2,36 & 1,10 & 0,77 & $\mathrm{Nd}$ & d 0,98 & 0,95 & 2,51 & 0,73 \\
\hline \multicolumn{11}{|c|}{ TERPENÓIDES } \\
\hline & 30 Limoneno $^{f}$ & 1037 & $\mathrm{Nd}$ & $\mathrm{Nd}$ & $\mathrm{Nd}$ & $\mathrm{Nd}$ & $\mathrm{Nd}$ & $\mathrm{Nd}$ & 0,12 & 0,89 \\
\hline & 39 Naftaleno ${ }^{f}$ & 1212 & 1,27 & $\mathrm{Nd}$ & 0,24 & $\mathrm{Nd}$ & d 1,46 & $\mathrm{Nd}$ & 0,23 & $\mathrm{Nd}$ \\
\hline \multicolumn{11}{|c|}{ COMPOSTOS BENZÊNICOS } \\
\hline & 2 Benzeno $^{f}$ & 602 & 3,51 & 20,20 & 5,49 & 0,40 & 21,23 & 7,32 & 7,83 & 8,64 \\
\hline & 11 Tolueno $^{f}$ & 769 & 1,39 & 2,39 & 0,96 & $\mathrm{Nd}$ & 0,32 & 1,79 & 2,56 & 0,36 \\
\hline & 15 Etilbenzeno $^{f}$ & 868 & 1,78 & 0,73 & 0,69 & $\mathrm{Nd}$ & 0,79 & 0,71 & 1,51 & 0,95 \\
\hline & 16 1,3-dimetilbenzeno ${ }^{f}$ & 877 & 1,90 & 1,99 & 1,69 & $\mathrm{Nd}$ & 1,19 & 2,53 & 1,56 & 1,22 \\
\hline & 17 1,4-dimetilbenzeno ${ }^{f}$ & 878 & 2,40 & $\mathrm{Nd}$ & $\mathrm{Nd}$ & $\mathrm{Nd}$ & 0,75 & $\mathrm{Nd}$ & 2,51 & 1,34 \\
\hline & 19 1,2-dimetilbenzeno ${ }^{f}$ & 901 & 1,75 & 0,58 & 0,66 & $\mathrm{Nd}$ & 0,90 & 0,75 & 1,18 & 1,15 \\
\hline & 21 1-metil-2-etilbenzeno ${ }^{\dagger}$ & 979 & 0,13 & $\mathrm{Nd}$ & $\mathrm{Nd}$ & $\mathrm{Nd}$ & $\mathrm{Nd}$ & $\mathrm{Nd}$ & 0,06 & 0,20 \\
\hline & 22 Benzaldeído ${ }^{\dagger}$ & 982 & 0,20 & $\mathrm{Nd}$ & 0,05 & $\mathrm{Nd}$ & 0,16 & $\mathrm{Nd}$ & 0,28 & 0,17 \\
\hline & 27 1,3,5-trimetilbenzeno ${ }^{f}$ & 1003 & 0,19 & $\mathrm{Nd}$ & 0,07 & $\mathrm{Nd}$ & 0,05 & $\mathrm{Nd}$ & 0,15 & 0,27 \\
\hline \multicolumn{11}{|c|}{ ALDEÍDOS } \\
\hline & 13 Hexanal $^{f}$ & 808 & 0,41 & $\mathrm{Nd}$ & $\mathrm{Nd}$ & $\mathrm{Nd}$ & 0,07 & $\mathrm{Nd}$ & 0,13 & $\mathrm{Nd}$ \\
\hline & 20 Heptanafl & 911 & 0,16 & $\mathrm{Nd}$ & $\mathrm{Nd}$ & $\mathrm{Nd}$ & $\mathrm{Nd}$ & $\mathrm{Nd}$ & 0,26 & $\mathrm{Nd}$ \\
\hline & 28 Octanal $^{f}$ & 1013 & 0,39 & $\mathrm{Nd}$ & $\mathrm{Nd}$ & $\mathrm{Nd}$ & $\mathrm{Nd}$ & $\mathrm{Nd}$ & 0,35 & $\mathrm{Nd}$ \\
\hline & 36 Nonanal $^{f}$ & 1114 & 1,12 & 0,16 & 0,34 & $\mathrm{Nd}$ & 0,78 & $\mathrm{Nd}$ & 1,17 & 0,63 \\
\hline & 40 Decanal $^{f}$ & 1216 & 0,15 & $\mathrm{Nd}$ & $\mathrm{Nd}$ & $\mathrm{Nd}$ & $\mathrm{Nd}$ & $\mathrm{Nd}$ & 0,10 & $\mathrm{Nd}$ \\
\hline & 51 Dodecanal $^{f}$ & 1419 & $\mathrm{Nd}$ & $\mathrm{Nd}$ & $\mathrm{Nd}$ & $\mathrm{Nd}$ & $\mathrm{Nd}$ & $\mathrm{Nd}$ & 0,05 & $\mathrm{Nd}$ \\
\hline & 58 Tetradecanal $^{f}$ & 1623 & 0,27 & 0,19 & 0,30 & 0,42 & 0,26 & $\mathrm{Nd}$ & 0,35 & 0,40 \\
\hline & 63 Pentadecanal $^{\dagger}$ & 1725 & 0,43 & 0,41 & 0,61 & 0,76 & 0,46 & 0,34 & 0,55 & 0,77 \\
\hline & 72 Hexadecanal $^{f}$ & 1828 & 5,40 & 9,39 & 15,42 & 22,30 & 8,53 & 7,51 & 10,55 & 14,57 \\
\hline & 83 Heptadecanal $^{f}$ & 1930 & 0,24 & 0,50 & 0,91 & 1,14 & 0,49 & 0,38 & 0,78 & 1,03 \\
\hline & 90 Octadecanal $^{\dagger}$ & 2032 & 4,10 & 5,49 & 8,75 & 12,12 & 3,95 & 3,89 & 7,59 & 8,28 \\
\hline & 32 (E) 2-Octenalf & 1071 & $\mathrm{Nd}$ & $\mathrm{Nd}$ & $\mathrm{Nd}$ & $\mathrm{Nd}$ & $\mathrm{Nd}$ & $\mathrm{Nd}$ & 0,09 & $\mathrm{Nd}$ \\
\hline & 37 (E) 2-nonenal ${ }^{f}$ & 1173 & $\mathrm{Nd}$ & $\mathrm{Nd}$ & $\mathrm{Nd}$ & $\mathrm{Nd}$ & $\mathrm{Nd}$ & $\mathrm{Nd}$ & 0,27 & $\mathrm{Nd}$ \\
\hline & 43 (E) 2-decenalf & 1275 & 0,18 & $\mathrm{Nd}$ & 0,46 & $\mathrm{Nd}$ & 0,11 & $\mathrm{Nd}$ & 0,56 & 0,24 \\
\hline & $48(\mathrm{E}, \mathrm{E})$ 2,4-decadienal ${ }^{\mathrm{f}}$ & 1337 & $\mathrm{Nd}$ & $\mathrm{Nd}$ & $\mathrm{Nd}$ & $\mathrm{Nd}$ & $\mathrm{Nd}$ & $\mathrm{Nd}$ & 0,33 & $\mathrm{Nd}$ \\
\hline & 49 (E) 2-undecenalf & 1378 & 0,13 & $\mathrm{Nd}$ & $\mathrm{Nd}$ & $\mathrm{Nd}$ & 0,10 & $\mathrm{Nd}$ & 0,58 & $\mathrm{Nd}$ \\
\hline & 71 (E) 2-hexadecenal ${ }^{f}$ & 1813 & $\mathrm{Nd}$ & $\mathrm{Nd}$ & 0,19 & 0,23 & $\mathrm{Nd}$ & $\mathrm{Nd}$ & 0,24 & 0,47 \\
\hline & 79 Octadecenal $^{h}$ & 1891 & 0,36 & 0,57 & 0,80 & 1,0 & 0,53 & 0,49 & 0,71 & $\mathrm{Nd}$ \\
\hline & 89 (Z) 9-octadecenal ${ }^{9}$ & 2010 & 0,59 & 1,31 & 0,83 & 3,57 & 1,93 & 2,01 & 1,96 & 2,19 \\
\hline \multicolumn{11}{|c|}{ ÁLCOOIS } \\
\hline & 24 1-octano-3-ol ${ }^{f}$ & 988 & 0,28 & $\mathrm{Nd}$ & 0,12 & $\mathrm{Nd}$ & 0,25 & $\mathrm{Nd}$ & 0,17 & 0,31 \\
\hline & 34 1-octanolf & 1079 & 0,06 & $\mathrm{Nd}$ & 0,10 & $\mathrm{Nd}$ & $\mathrm{Nd}$ & $\mathrm{Nd}$ & 0,29 & 0,19 \\
\hline & 59 1-tetradecanol ${ }^{f}$ & 1662 & $\mathrm{Nd}$ & $\mathrm{Nd}$ & $\mathrm{Nd}$ & $\mathrm{Nd}$ & $\mathrm{Nd}$ & $\mathrm{Nd}$ & 0,18 & $\mathrm{Nd}$ \\
\hline & 66 1-pentadecanol ${ }^{\dagger}$ & 1789 & 0,19 & 0,55 & 0,54 & 0,73 & 1,32 & $\mathrm{Nd}$ & 0,55 & 0,59 \\
\hline & 87 1-heptadecanol ${ }^{\dagger}$ & 1993 & $\mathrm{Nd}$ & $\mathrm{Nd}$ & 0,22 & 0,36 & 0,25 & $\mathrm{Nd}$ & 0,21 & $\mathrm{Nd}$ \\
\hline & 31 2-ethyl-1-hexanolf & 1038 & 7,55 & 2,41 & $\mathrm{Nd}$ & 0,40 & 2,97 & 3,80 & 0,06 & $\mathrm{Nd}$ \\
\hline & 46 Álcool $^{\text {h }}$ & 1312 & $\mathrm{Nd}$ & $\mathrm{Nd}$ & $\mathrm{Nd}$ & $\mathrm{Nd}$ & $\mathrm{Nd}$ & $\mathrm{Nd}$ & 0,32 & 0,40 \\
\hline & 84 Álcool $^{\mathrm{h}}$ & 1936 & 0,11 & 0,29 & 0,19 & 0,19 & 0,10 & 3,89 & 0,20 & 0,24 \\
\hline & 07 Álcool $^{\text {h }}$ & & 4,47 & 4,42 & 0,33 & 0,20 & $\mathrm{Nd}$ & 1,39 & 0,11 & 0,54 \\
\hline \multicolumn{11}{|c|}{ ÉSTERES } \\
\hline & 42 Etil no & 1268 & 0,14 & $\mathrm{Nd}$ & 0,09 & $\mathrm{Nd}$ & 0,11 & $\mathrm{Nd}$ & 0,13 & 0,15 \\
\hline & 47 Metil decanoato ${ }^{f}$ & 1328 & 0,19 & $\mathrm{Nd}$ & $\mathrm{Nd}$ & 0,08 & 0,23 & 0,42 & 0,14 & 0,21 \\
\hline
\end{tabular}




\begin{tabular}{|c|c|c|c|c|c|c|c|c|c|c|}
\hline \multirow[t]{2}{*}{ No } & \multirow[t]{2}{*}{ Classe e Nome do Composto ${ }^{a}$} & \multirow[t]{2}{*}{$\mathrm{IRL}^{\mathrm{b}}$} & \multicolumn{3}{|c|}{ Abundância Relativac $^{c}$} & 220 dias & \multicolumn{2}{|c|}{265 dias } & \multicolumn{2}{|c|}{310 dias } \\
\hline & & & $\% 1 \mathrm{C} \%$ & 611 & $\% 2 \mathrm{C}$ & $\% 21$ & $\% 3 \mathrm{C}$ & $\% 31$ & $\% 4 \mathrm{C}$ & $\% 41$ \\
\hline \multicolumn{11}{|c|}{ CETONAS } \\
\hline & 452-undecanona ${ }^{f}$ & 1303 & 0,14 & $\mathrm{Nd}$ & 0,1 & 0,07 & 0,18 & $\mathrm{Nd}$ & 0,70 & 0,27 \\
\hline & 542-tridecanona ${ }^{f}$ & 1503 & 0,47 & $\mathrm{Nd}$ & 0,0 & 90,11 & 0,11 & $\mathrm{Nd}$ & 0,48 & 0,18 \\
\hline & 622-pentadecanona ${ }^{f}$ & 1705 & 1,39 & 0,59 & 1,1 & $2 \quad 0,96$ & 1,14 & $4 \quad 0,75$ & 1,78 & 0,89 \\
\hline & 702-hexadecanona ${ }^{f}$ & 1808 & 0,16 & $\mathrm{Nd}$ & 0,0 & $7 \quad 0,10$ & $\mathrm{Nd}$ & $\mathrm{Nd}$ & 0,19 & 0,16 \\
\hline & 822-heptadecanona ${ }^{f}$ & 1909 & 1,10 & 0,66 & 0,8 & 30,78 & 0,72 & 20,79 & 1,63 & 1,35 \\
\hline & 99 Cetona aliciclica ${ }^{\mathrm{h}}$ & 2173 & $\mathrm{Nd}$ & $\mathrm{Nd}$ & 0,7 & 0,49 & 0,97 & $7 \quad 0,75$ & 0,32 & $\mathrm{Nd}$ \\
\hline & 552-metil ciclotridecanona ${ }^{f}$ & 1573 & 0,23 & $\mathrm{Nd}$ & 0,2 & 20,13 & 0,18 & $\mathrm{Nd}$ & 0,14 & 0,18 \\
\hline \multicolumn{11}{|c|}{ COMPOSTOS SULFURADOS } \\
\hline & 23Etil 1-metiletil disulfito ${ }^{f}$ & 984 & 0,86 & 0,39 & 0,4 & $\mathrm{Nd}$ & 0,69 & 90,44 & 0,17 & 0,99 \\
\hline & 29Bis(1-metiletil)disulfito ${ }^{\dagger}$ & 1030 & 0,64 & 0,31 & 0,3 & $\mathrm{Nd}$ & 0,62 & 20,62 & 0,15 & 0,98 \\
\hline & 41 Dipropiltrisulfito $^{\dagger}$ & 1250 & $\mathrm{Nd}$ & $\mathrm{Nd}$ & N & $\mathrm{Nd}$ & $\mathrm{Nd}$ & $\mathrm{Nd}$ & 0,17 & $\mathrm{Nd}$ \\
\hline & 252-pentilfurano ${ }^{\dagger}$ & 995 & 0,26 & $\mathrm{Nd}$ & Nc & $\mathrm{Nd}$ & 0,39 & $\mathrm{Nd}$ & 0,18 & 0,02 \\
\hline \multicolumn{11}{|c|}{ OUTROS COMPOSTOS } \\
\hline & $\begin{array}{l}\text { 78Bis(2-metilpropil)-1,2-benzene ácido } \\
\text { dicarboxilíco }\end{array}$ & 1881 & 3,46 & 0,87 & 0,3 & 30,42 & 0,54 & $4 \quad 0,49$ & 0,89 & 2,21 \\
\hline & 98Bis(2-etilhexil)ester ácido hexanodioico & 2162 & 0,77 & 0,70 & 2,7 & 8,52 & 0,31 & 1,70 & 4,41 & 6,67 \\
\hline & 75Anthracene & 1846 & 0,38 & $\mathrm{Nd}$ & 0,1 & $\mathrm{Nd}$ & 0,65 & $\mathrm{Nd}$ & 0,10 & 0,22 \\
\hline
\end{tabular}

a Compostos foram identificados através dos espectro de massa da referência "The Eight Peak Index [1].

${ }^{\mathrm{b}}$ IRL - Indice de Retenção Linear.

'Abundância relativa em \%, calculada como 100 x área do pico/área total do cromatograma

${ }^{d} \mathrm{ND}$ - não identificado.

e Número total de compostos identificados em cada classe

iComposto cujo espectro de massa e IRL concordam com aqueles de compostos autênticos analisados em condições similares.

g espectros de massas concordam com "The Eight Peak Index"

h interpretação dos espectros de massa e comparação com aqueles de compostos relacionados

I - Inteiro

alifáticos sulfurados. Conforme apresentado na Tabela 2, os hidrocarbonetos (alifáticos e aromáticos) apresentaram o maior somatório de abundância relativa em todos os extratos caprinos pesquisados, seguido pelos aldeídos e/ou compostos benzênicos e/ou álcoois.

Dos cento e oito voláteis listados, doze apresentaram abundância relativa consideráveis, isto é $>1,5 \% \mathrm{em}$ praticamente todas as amostras. Destes seis eram hidrocarbonetos ( 4 alcanos e 2 alcenos), dois compostos benzênicos, três aldeídos e um ácido. Analisando-se os indices de abundância dos diferentes compostos nas várias amostras observou-se que o composto que apresentou maior indice foi o hexadecanal, seguido pelo benzeno, heptano e octadecanal (Tabela 1).

Hidrocarbonetos alifáticos, aldeídos, alcoois e cetonas têm sido reportados freqüentemente como os principais voláteis do perfil aromático de carne cozida, não constituindo assim surpresa sua abundância entre os voláteis da carne caprina. A ausência de ácidos carboxílicos nos extratos caprinos foi justificada pelo baixo teor de gordura da carne caprina, cujo teor variou de 1,62 a 5,42\% $[11,13,14]$. MILLER, FIELD \& AGBOOLA [15] reportaram que os ácidos graxos de cadeira ramificada ocorrem em maiores concentrações na gordura subcutânea quando comparada com a gordura muscular de ovinos.

Os voláteis identificados nos extratos de carne caprina constituem compostos formados a partir da degradação térmica dos fosfolipídeos, triglicerídeos e áci- dos graxos presentes nas frações gordurosas da carne caprina. Apenas os compostos sulfurados provavelmente foram formados envolvendo reações de Maillard entre os aminoácidos sulfurados (a exemplo da cisteína) e a ribose $[10,17,18]$. Vale citar que todos os compostos identificados já foram previamente reportados em perfis aromáticos de carne cozida de diferentes espécies de animais, principalmente em carne bovina, e seus precursores e mecanismos de formação foram revistos em diversas publicações $[5,9,16,17,18,20]$.

Comparando-se o número total de voláteis (análise qualitativa) presente nos extratos de caprinos castrados e inteiros (Tabela 2), observa-se uma predominância de voláteis nos extratos de carne de caprinos castrados para todas as dez classes de compostos identificados. Uma tendência semelhante pode ser observada na análise quantitativa, ou seja de abundância relativa total, na qual os extratos de animais castrados apresentaram abundância relativa dos picos superiores aos extratos de animais inteiros para as classes de hidrocarbonetos alicíclicos, terpenoídes, compostos benzênicos, cetonas e compostos sulfurados. Apenas a classe dos aldeídos teve maior abundância relativa dos picos em extratos de caprinos inteiros. Para as classes de álcoois e ésteres esta tendência não ficou muito clara. A maior concentração de voláteis formados em extratos de caprinos castrados resultou provavelmente do fato de que a carne de animais castrados também apresentou maior teor de gordura [11, 13, 14] CAPORASO et al. [3] consideram os compostos aldeídicos 
TABELA 2. Abundância relativa das principais classes de compostos voláteis identificados em extratos de carne caprina de animais inteiros e castrados abatidos com diferentes idades.

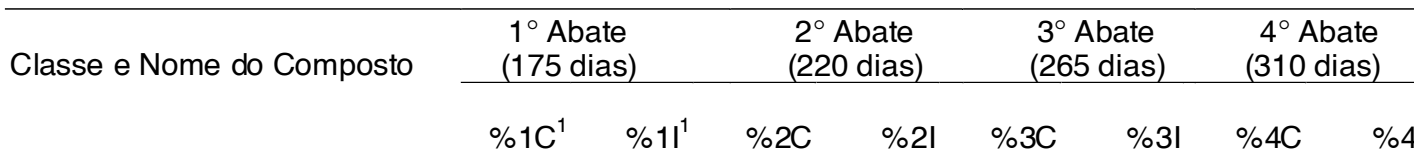

Hidrocarbonetos Alifáticos (41)

Número Total Presente

Área Relativa Total (ART)

$\begin{array}{rrrrrrrr}33 & 26 & 38 & 27 & 36 & 25 & 38 & 31 \\ 28,79 & 40,38 & 37,28 & 41,67 & 35,74 & 44,57 & 28,29 & 31,94\end{array}$

Hidrocarbonetos Aliciclícos (12)

Número Total Presente

Área Relativa Total (ART)

$\begin{array}{rrrrrrrr}10 & 8 & 11 & 7 & 11 & 9 & 10 & 9 \\ 19,09 & 4,49 & 15,4 & 2,79 & 8,38 & 12,65 & 13,01 & 7,51\end{array}$

Terpenoides (2)

Número Total Presente

Área Relativa Total (ART)

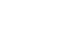

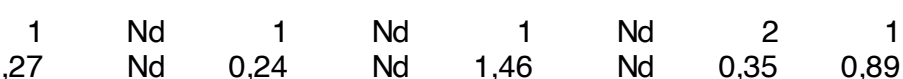

Compostos Benzênicos (9)

Número Total Presente

Área Relativa Total (ART)

13,25

Aldeídos (19)

Número Total Presente

Área Relativa Total (ART)

$\begin{array}{rrrrrrrr}14 & 8 & 10 & 8 & 11 & 6 & 19 & 9\end{array}$

Álcoois (9)

Número Total Presente

Área Relativa Total (ART)

$\begin{array}{rrrrrrrr}6 & 4 & 6 & 5 & 5 & 3 & 9 & 6 \\ 12,66 & 7,67 & 1,5 & 1,88 & 4,89 & 9,08 & 2,09 & 2,27\end{array}$

Ésteres (2)

Número Total Presente

Área Relativa Total (ART)

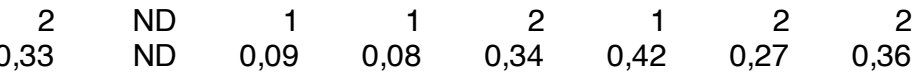

Cetonas (7)

Número Total Presente

Área Relativa Total (ART)

$\begin{array}{rrrrrrrr}6 & 2 & 7 & 7 & 6 & 3 & 7 & 6 \\ 3,49 & 1,25 & 3,2 & 2,64 & 3,3 & 2,29 & 5,24 & 3,03\end{array}$

Compostos Sulfurados (4)

Número Total Presente

Área Relativa Total (ART)

$\begin{array}{rrrrrrrr}3 & 2 & 2 & \text { ND } & 3 & 2 & 4 & 3 \\ 1,76 & 0,7 & 0,76 & \text { ND } & 1,7 & 1,06 & 0,67 & 1,99\end{array}$

Outros Compostos (3)

Número Total Presente

Área Relativa Total (ART)

${ }^{1} \mathrm{C}$ - castrado - inteiro

alifáticos e alguns compostos cetônicos como possiveis contribuídores do aroma de carne de ovelha.

O número total de voláteis e a abundância relativa das diferentes classes de compostos não foram claramente afetados pelo fator idade de abate, uma vez que não foi observado aumento ou diminuição destes parâmetros com o aumento da idade de abate (Tabelas 1 e 2).

\section{4 - CONCLUSÕES}

- Os extratos de carne caprina de animais castrados apresentaram maior número e abundância relativa de voláteis. O fator idade de abate não afetou o número total de voláteis e a abundância relativa das diferentes classes de compostos aromáticos dos extratos de carne caprina.

\section{5 - REFERÊNCIAS BIBLIOGRÁFICAS}

[1] ANON. Eight Peak Index of Mass Spectra, $3^{\text {rd }}$ edn. , MSDC, Aldermaston, UK. 1983.

[2] BRENNAND, C. P. Factors affecting contributions of volatile branched-chain fatty acids to the species related flavors of lamb and mutton. PhD thesis, University of Wisconsin, Madison, WI, USA. 1989.

[3] CAPORASO, F.; SINK, J.D.; DIMICK, P. S.; MUSSINAN, C.J. \& SANDERSON, A. Volatile flavor constituents of ovine adipose tissue. J. Agric. Food Chemistry, v. 25, p. 12301233, 1977. 
[4] CRAMER, D.A. Chemical compounds implicated in lamb flavour. Food Technology, v. 37, p. 249-257, 1983.

[5] DWIVEDI, B. K. Meat flavor. CRC Crit. Ver. Food Technology, v. 5, p. 487-535, 1975

[6] HA, J. K. \& LINDSAY, R. C. Volatiles alkylphenols and thiophenol in species-related characterising flavors of red meats. J. Food Science, v. 56, p. 1197-1202, 1991.

[7] KIM, J. \& LINDSAY, R.C. Volatile alkylphenols and thiophenol in species-related characterising flavors of red meats. J. Food Science, v. 56, n. 5, p. $1197-$ 1202. 1991.

[8] LIKENS, S. T. \& NICKERSON, G. B. Detection of certain Hop oil constituents in brewing products. Proc. Am. Soc. Brew. Chem., v. 5, p. 13-19, 1964.

[9] MACLEOD, G.M. \& SEYYEDAIN-ARDEBILI, M. Natural and simulated meat flavors (with particular reference to beef). CRC Crit. Ver. Food Sci. Nutr., v. 14, p. 309-437, 1981.

[10] MADRUGA, M. S. Studies on some factors affecting meat flavour formation. PhD thesis, University of Reading, Reading, England, UK. 1994.

[11] MADRUGA, M.S.; ARRUDA, S.G.B. \& NASCIMENTO, J.A. Castration and slaughter age effects on nutritive value of the "mestiço" goat meat. Meat Science, v. 52, p. 119-125, 1999.

[12] MADRUGA, M.S.; ARRUDA, S.G.B.; Narain, N. \& Souza, J.G. Castration and slaughter age effects on panel assessment and aroma compounds of the "mestiço" goat meat. Meat Science, v. 56, p. 117-125, 2000.

[13] MADRUGA, M.S.; NARAIN, N,; SOUZA, J.G. Castration and slaughter age effects on fat components of "Mestiço" goat meat. Small Ruminant Research. v. 42, p. 77-82, 2001.

[14] MADRUGA, M.S.; NARAIN, N. ; ARRUDA, S.G.B. \& Souza, J.G. Influência da idade de abate e da castração nas qualidades físico-químicas, sensoriais e aromáticas da carne caprina. Revista Brasileira de Zootecnia, v. 31, p. 1562-1570, 2002.
[15] MILLER, G.J.; FIELD, R.A.\& AGBOOLA, H.A. Lipids in subcutaneous tissues and Longissmus muscles of feedlot and grassfed ewes. J. Food Quality, v. 9, p. 39-47, 1986.

[16] MOTTRAM, D.S. Meat. In Volatile compounds in Foods and Beverages. Ed. H. Maarse. Marcel Dekker, New York, p. 107-177. 1991.

[17] MOTTRAM, D. S. Meat flavour: a review. In Understanding Natural Flavours, ed. J. R. Piggott \& A. Patterson. Blackie, Glasgow, p. 140-163. 1994.

[18] MOTTRAM, D.S. Flavour formation in meat and meat products: a review. Food Chemistry, v. 62, n. 4, p. 415424, 1998.

[19] ROUSSEL-AKIM, S.; YOUNG, O. A. \& BERDAGUÉ. Diet and growth in panel assessment of sheepmeat odour and flavour. Meat Science, v. 45, n. 2, p. 169-181, 1997.

[20] SHAHIDI, F.; RUBIN, L.J. \& D'SOUZA, L.A. Meat flavor volatiles: a review of the composition, techniques of analysis, sensory evaluation. CRC Crit. Rev. Food Science and Nutrition, v. 24, p. 141-243. 1986.

[21] SINK, J. D. Factors influencing the flavour of muscle foods. J. Food Science, v. 44, p. 1-5, 1979.

[22] SUTHERLAND, M.M. \& AMES, J.M. The effect of castration on the headspace aroma components of cooked lamb. J. Sci. Food Agriculture, v. 69, p. 403-413. 1995.

[23] YOUNG, O. A.; BERDAGUÉ, C. V.; ROUSSET-AKRIM, S.; THERIEZ, M. Fat-borne volatiles and sheepmeat odour. Meat Science, v. 45, n. 2, p. 183-200, 1997.

[24] WONG, E., NIXON, L. N. \& JOHNSON, C. B. Volatile medium chain fatty acids and mutton flavor. J. Agric. Food Chemistry, v. 23, p. 495-498, 1975.

\section{6 - AGRADECIMENTOS}

Os autores agradecem ao CNPq pelo apoio financeiro e pelas bolsas concedidas. Ao Professor Narendra Narain, do Departamento de Tecnologia Química e de Alimentos (DTQA/UFPB) pela realização das análises de voláteis $(\mathrm{CG} / \mathrm{EM})$. 